\title{
Rapid identification of Vibrio cholerae serotype 01 from primary isolation plates by a coagglutination test
}

\author{
M. RAHMAN, D. A. SACK*, A. WADOOD, M. YASMIN and A. LATIF
}

Laboratory Sciences Division, International Centre for Diarrhoeal Disease Research, Bangladesh, GPO Box 128 , Dhaka-1000, Bangladesh, and "Division of Geographic Medicine, Johns Hopkins University, Baltimore, MD 21205, USA

\begin{abstract}
Summary. A coagglutination test was developed for identifying suspected colonies of Vibrio cholerae serotype $\mathrm{O} 1$ directly from primary isolation plates. Visible agglutination occurs when $V$. cholerae $\mathrm{Ol}$ antibody attached to cell-wall protein A of Staphylococcus aureus reacts with its homologous antigen. From 314 faecal samples from clinically suspected cases of cholera, 210 colonies from thiosulphate citrate bile salts sucrose (TCBS) agar and 222 colonies from taurocholate tellurite gelatin (TTG) agar were tested as suspect $V$. cholerae. In each case 204 isolates were identified as $V$. cholerae $\mathrm{O} 1$ by conventional methods and also gave positive results for $V$. cholerae $\mathrm{O} 1$ in the coagglutination test; with one partial exception, no other colonies tested gave positive results. The coagglutination test is simple and inexpensive and provides a result $24 \mathrm{~h}$ earlier than conventional methods.
\end{abstract}

\section{Introduction}

Cholera is still a major public health problem in many developing countries (Samadi et al., 1983). Early diagnosis is essential so that effective therapy and epidemiological measures can be initiated. Vibrio cholerae serotype $\mathrm{Ol}$ is the causative agent (Farmer et al., 1985) and conventional laboratory diagnosis depends upon isolation by culture and identification by biochemical and serological tests (Farmer et al., 1985). These procedures are expensive and normally take about 2 days to obtain a result. Moreover, the limited facilities available for expensive biochemical tests in small laboratories in many developing countries make the identification of this organism difficult in such conditions. Morris et al. (1979) described a slide agglutination test for the identification of $V$. cholerae $\mathrm{O} 1$, by testing colonies taken directly from thiosulphate citrate bile-salts sucrose (TCBS) agar and taurocholate tellurite gelatin (TTG) agar with specific antiserum, in order to reduce identification time and costs. However, although the less frequently used TTG agar was suitable for this purpose, $V$. cholerae $\mathrm{O} 1$ colonies from the more widely used TCBS agar gave negative or doubtful results in the majority of

Received 19 Feb. 1988; revised version accepted 20 July 1988. cases (Monsur, 1961; Kobayashi et al., 1963; Morris et al., 1979).

Coagglutination, another rapid serological test, is used to detect antigens of the causative bacteria of pneumonia, meningitis and typhoid in clinical specimens (Kronvall, 1973; Thirumoorthi and Dajani, 1979; Sivadasan et al., 1984; Fung and Tilton, 1985). Formaldehyde-stabilised, heattreated cells of Staphylococcus aureus Cowan 1 (ATCC 12598), which contains protein A in its cell wall, are treated with high-titre specific antiserum. Protein A binds IgG by the Fc fragment and, in the presence of homologous antigen, the antibodycoated staphylococcal-cell reagent produces visible agglutination. We have previously described the use of a coagglutination test with 4-h faecal enrichment cultures for the rapid diagnosis of cholera (Rahman et al., 1987). In this paper we describe its application to the identification of colonies taken from primary isolation plates to facilitate rapid screening of faecal samples for $V$. cholerae $\mathrm{O} 1$.

\section{Materials and methods}

\section{Detection of $V$. cholerae in faeces}

Faecal samples from 314 suspected cases of cholera were cultured on TCBS (Eiken Chemical Co. Ltd, Tokyo, 
Japan) and TTG agar (Monsur, 1961). V. cholerae O1 was identified by conventional biochemical and serological tests (Farmer et al., 1985).

\section{Preparation of coagglutination reagents}

A modification of the method of Kronvall (1973) was used to prepare coagglutination reagents (Rahman et al., 1987). High-titre $V$. cholerae $O 1$ antiserum was prepared by multiple inoculation of rabbits with live $V$. cholerae O1. S. aureus Cowan 1, grown overnight in trypticase soya broth with shaking at $36^{\circ} \mathrm{C}$, was washed with phosphate-buffered saline (PBS; $0.03 \mathrm{M}$ phosphate, $0.12 \mathrm{M} \mathrm{NaCl}, \mathrm{pH} \mathrm{7.3)}$ and stabilised with formaldehyde $0.5 \%$ in PBS. The cells were heated for $1 \mathrm{~h}$ at $80^{\circ} \mathrm{C}$, washed and $1 \mathrm{ml}$ of a $10 \% \mathrm{v} / \mathrm{v}$ suspension of cells in PBS was mixed with $0.2 \mathrm{ml}$ of $V$. cholerae $\mathrm{O} 1$ antiserum and incubated for $3 \mathrm{~h}$ at room temperature $\left(25^{\circ} \mathrm{C}\right)$ with shaking. The antibody-coated staphylococci were washed with PBS and resuspended at $2 \% \mathrm{v} / \mathrm{v}$ in PBS containing sodium azide $0.1 \%$ to form the coagglutination reagent. A formaldehyde-stabilised, heat-treated $2 \% \mathrm{v} / \mathrm{v}$ suspension of staphylococci in PBS containing sodium azide $0.1 \%$ was used as a negative control reagent. Reagents were stored at $4^{\circ} \mathrm{C}$ until used. $V$. cholerae $\mathrm{O} 1$ lipopolysaccharide (List Biological Laboratory, USA) was used as a positive antigen control; $0.5 \mathrm{mg} / \mathrm{ml}$ in PBS gave easily visible agglutination.

\section{Slide coagglutination test}

The reagents were brought to room temperature and shaken to obtain a uniform bacterial suspension. Four rectangles were made with a wax pencil on a glass slide. In the first two rectangles, a suspension of bacterial cells in normal saline was prepared from suspected $V$. cholerae colonies on the TCBS agar plate, i.e., sucrose-positive, yellow colonies. In the other two rectangles, a suspension was made with suspected colonies from the TTG agar plate, i.e., gelatinase-positive colonies (halo-positive). One drop of coagglutination reagent was added to the first and third rectangles and one drop of the negative control reagent to the second and fourth rectangles and the drops were mixed well with sticks. After rotation by hand for $2 \mathrm{~min}$, the slides were examined against a dark background with a viewing lamp. The coagglutination test was considered positive if macroscopic agglutination was seen with coagglutination reagent but not with the control reagent.

\section{Results}

Suspected colonies of $V$. cholerae from the primary isolation plates were screened by conventional methods and by the coagglutination test. The results are shown in the table. $V$. cholerae $\mathrm{O} 1$ was detected in $204(65 \%)$ of 314 faecal samples by conventional methods. The coagglutination test identified all 204 of these isolates as $V$. cholerae $\mathrm{O} 1$, with colonies from both TCBS and TTG agar plates; there was no difference in the results obtained from either plate. The suspected colonies of $V$. cholerae from the six TCBS agar plates that gave negative results in the coagglutination test were all identified as other than $V$. cholerae $\mathrm{Ol}$ by conventional methods (table). With TTG medium, there were suspect colonies from 18 plates that proved to be other than $V$. cholerae $\mathrm{O} 1$ by conventional methods and 17 of these gave negative coagglutination reactions, the other giving a weakly positive result although it was identified as $V$. mimicus by conventional methods (table). None of the strains identified as $V$. cholerae $\mathrm{O} 1$ gave negative or even weakly positive coagglutination reactions; all gave moderate or strong (some clumping) reactions with colonies from either isolation medium; four TCBS isolates and 30 TTG isolates gave very strong reactions (coarse clumping).

\section{Discussion}

The development of more rapid, specific and economic means of detection of diarrhoeal pathogens is desirable, in order to provide the early aetiological diagnosis needed for initiation of proper treatment and public health measures. The

Table. Comparison of results obtained with suspected colonies* of $V$. cholerae in conventional tests and in the coagglutination test

Identity and (number) of isolates determined by

\begin{tabular}{|c|c|c|}
\hline $\begin{array}{l}\text { Number of speci- } \\
\text { mens with suspected } \\
\text { colonies on the indi- } \\
\text { cated medium }\end{array}$ & Conventional methods & $\begin{array}{l}\text { Coagglutina- } \\
\text { tion- } \\
\text { test }\end{array}$ \\
\hline \multicolumn{3}{|l|}{ TCBS agar } \\
\hline 210 & $\begin{array}{l}\text { V. cholerae } \mathrm{O} 1 \text { (204) } \\
\text { V. fluvialis (4) } \\
\text { V. cholerae non } \mathrm{O} 1(2)\end{array}$ & $\begin{array}{l}+(204) \\
-(4) \\
-(2)\end{array}$ \\
\hline \multicolumn{3}{|l|}{ TTG agar } \\
\hline 222 & $\begin{array}{l}\text { V. cholerae O1 (204) } \\
V . \text { fluvialis (4) } \\
V . \text { cholerae non } \mathrm{O} 1 \text { (2) } \\
\text { V. parahaemolyticus (3) } \\
\text { V. mimicus (3) } \\
\text { Aeromonas hydrophila (3) } \\
\text { A. sobria (3) }\end{array}$ & $\begin{array}{l}+(204) \\
-(4) \\
-(2) \\
-(3) \\
-(2) \dagger \\
-(3) \\
-(3)\end{array}$ \\
\hline
\end{tabular}

$+=$ Positive result $;-=$ negative result .

* Sucrose-positive yellow colonies from TCBS and gelatinase positive colonies (with halo) from TTG agar.

† One strain from TTG agar gave a weak reaction in the coagglutination test. 
isolation and identification of $V$. cholerae $\mathrm{O} 1$ from faeces by conventional culture methods is timeconsuming, laborious and expensive. The coagglutination test described here is performed directly from the primary isolation plates and can, therefore, be used to diagnose cholera one day earlier than hitherto. The sensitivity and specificity of the test were both equal to those obtained with conventional methods. Furthermore, no cross-reactions were observed in preliminary slide coagglutination tests with colonies of commonly isolated species of Vibrionaceae held in our stock culture. Unfortunately, the number of $V$. cholerae non $\mathrm{Ol}$ and other vibrios isolated in the study was small.

$V$. cholerae $\mathrm{O} 1$ was identified by the coagglutination test in 18-24 h with colonies from either TCBS or TTG agar. The preparation of coagglutination reagents is simple and whole $V$. cholerae $\mathrm{O} 1$ hyperimmune serum can be used to coat the staphylococci which form the basis of the test. Because only $0.2 \mathrm{ml}$ of antiserum is required for $5 \mathrm{ml}$ of coagglutination reagent, the test conserves antiserum, allowing about 25 times as many tests for a given volume of antiserum as does the conventional slide agglutination method. It is easy to perform, requires few materials and does not require highly trained or skilled laboratory workers. The results of the coagglutination test were easily readable, producing clear aggregation on the test slide in all positive samples that we examined.

The test has several potentially useful advantages

\section{REFERENCES}

Farmer J J, Hickman-Brenner F W, Kelly M T 1985 Vibrio. In: Lennette $\mathrm{E} \mathrm{H}$ et al. (eds) Manual of clinical microbiology 4th edn. American Society for Microbiology, Washington DC, pp 282-301.

Fung F C, Tilton R C 1985 Detection of bacterial antigens by counterimmunoelectrophoresis, coagglutination, and latex agglutination. In: Lennette $\mathrm{E} \mathrm{H}$ et al. (eds) Manual of clinical microbiology 4th edn. American Society for Microbiology, Washington DC, pp 883-890.

Kobayashi T, Enomoto S, Sakazaki R, Kuwahara S 1963 A new selective isolation medium for the Vibrio group; on a modified Nakanishi's medium (TCBS agar medium). Japanese Journal of Bacteriology 18: 387-392.

Kronvall G 1973 A rapid slide-agglutination method for typing pneumococci by means of specific antibody absorbed to protein A-containing staphylococci. Journal of Medical Microbiology 6: 187-190.

Monsur K A 1961 A highly selective gelatin-taurocholate- and applications. Firstly, as already indicated, it can be used to confirm the diagnosis of cholera earlier than by conventional methods. Secondly, as a reliable test, it could eliminate the need for expensive and tedious routine biochemical tests. Thirdly, either of the two isolation media (TCBS and TTG agar) recommended by the World Health Organisation (1974) can be used for the test, although TCBS has special advantages. As few Vibrio spp. produce yellow colonies on TCBS, the possibility of a false identification is very low (table), giving this medium an advantage over others. Moreover, TCBS agar does not require sterilisation during preparation; hence the coagglutination test with TCBS agar is practicable for field use, as well as for use in small laboratories with minimal facilities. Finally, identification of $V$. cholerae $\mathrm{O} 1$ by this method would allow antibiotic susceptibility tests to be performed earlier, with evident potential clinical benefits. Considering these advantages, together with its high sensitivity and specificity, we recommend the routine use of the rapid coagglutination test described here for the laboratory diagnosis of cholera.

We thank all Hospital Physicians of ICDDR,B for their assistance in providing clinical samples, Dr I. Ciznar for reviewing the manuscript and $\mathrm{MrS}$. K. Sarker for his secretarial assistance. This work was supported by a grant from the United Nations Development Program and the World Health Organisation.

tellurite medium for isolation of Vibrio cholerae. Transactions of the Royal Society of Tropical Medicine and Hygiene $\mathbf{5 5}$. 440-442.

Morris G K, Merson M H, Huq I, Kibrya A K M G, Black R 1979 Comparison of four plating media for isolating Vibrio cholerae. Journal of Clinical Microbiology 9: 79-83.

Rahman M, Sack D A, Mahmood S, Hossain A 1987 Rapid diagnosis of cholera by coagglutination test using 4-h fecal enrichment cultures. Journal of Clinical Microbiology 25: 2204-2206.

Samadi A R et al. 1983 Classical Vibrio cholerae biotype displaces El Tor in Bangladesh. Lancet 1: 805-807.

Sivadasan K, Kurien B, John T J 1984 Rapid diagnosis of typhoid fever by antigen detection. Lancet 1: 134-135.

Thirumoorthi M C, Dajani A S 1979 Comparison of staphylococcal coagglutination, latex agglutination and counterimmunoelectrophoresis for bacterial antigen detection. Journal of Clinical Microbiology 9, 28-32.

World Health Organization 1974 Guidelines for the laboratory diagnosis of cholera. World Health Organization, Geneva. 